\title{
Labyrinthe
}

34 | 2010 (1)

Comment peut-on être systématique?

\section{Notes éparses sur ce que signifie être systématique aujourd'hui}

\section{Diogo Sardinha}

\section{(2) OpenEdition \\ 1 Journals}

\section{Édition électronique}

URL : http://journals.openedition.org/labyrinthe/4061

DOI : $10.4000 /$ labyrinthe.4061

ISSN : 1950-6031

Éditeur

Hermann

\section{Édition imprimée}

Date de publication : 1 février 2010

Pagination : 119-128

ISBN : 978-2-7056-6984-3

\section{Référence électronique}

Diogo Sardinha, « Notes éparses sur ce que signifie être systématique aujourd'hui », Labyrinthe [En ligne], 34 | 2010 (1), mis en ligne le 01 février 2012, consulté le 03 mai 2019. URL : http:// journals.openedition.org/labyrinthe/4061 ; DOI : 10.4000/labyrinthe.4061 


\title{
Notes éparses sur ce que signifie être systématique aujourd'hui
}

\author{
Diogo SARDINHA \\ diogo_pt@hotmail.com
}

\section{À Monsieur Alain Kirili, sculpteur}

Ce texte est né d'un étonnement philosophique devant le vocabulaire et le discours de la science. Ajoutons tout de suite qu'on s'étonne le plus souvent non pas de ce qui est caché et secret, mais de ce qui est nettement perceptible. Dans le cas présent, ce qui m'a étonné a été l'intervention du biologiste moléculaire, professeur au Collège de France, François Gros, lors du colloque Alexander von Humboldt de 2008, à Paris ${ }^{1}$. Il était question, à un moment de son exposé, de la systématicité dans la science contemporaine. M. Gros parlait alors de scientifiques systématiciens comme Edward Osborne Wilson et Carl Woese, de leur précurseur Linée et des classements que celui-ci proposa, et faisait allusion aux systèmes et sous-systèmes moléculaires que l'on connaît aujourd'hui. Cette terminologie, aussi courante soit-elle dans un vaste domaine du savoir, contraste fortement avec celle dont se servent les philosophes depuis quelques décennies. La philosophie, en effet, s'est depuis un bon moment départie des systèmes, surtout depuis qu'elle a fustigé le travail de quelques penseurs majeurs du XIX ${ }^{\mathrm{e}}$ siècle, de Hegel à Comte et Marx. La modernité - époque dont on sait qu'elle a commencé au début du $\mathrm{XIX}^{\mathrm{e}}$ siècle, mais dont on n'a pas encore réussi à déterminer avec exactitude quand elle s'achève - a eu l'ambition de rendre compte des lois du monde et de l'histoire par les concepts les plus abstraits, prétendument susceptibles d'embrasser une logique du tout. Ses critiques ont soit explicitement abandonné, comme Nietzsche, soit discrètement laissé en suspens, comme le structuralisme, le vocabulaire du système et de la systématicité. D'où ma surprise à entendre parler de systèmes et

1. «La diversité biologique », communication au Collège Humboldt « La fascination de la planète », dirigé par Damien Erhardt et Soraya Nour, Goethe-Institut, 5-8 nov. 2008. 


\section{Labyrinthe, $n^{\circ} 34$}

de sous-systèmes, d'autant plus que, dans mon esprit de philosophe, de système il n'y en a qu'un, dominé par une logique d'ensemble, certes compatible avec un ensemble et ses parties, mais des parties dont on ne dirait qu'improprement qu'elles forment « des sous-systèmes ».

Les scientifiques qui ont l'habitude de ce vocabulaire et de la façon dont il leur permet d'organiser les objets dont ils font l'expérience diront que ma surprise est bien naïve. Même les philosophes répliqueront que le but des systèmes n'est pas nécessairement d'embrasser la totalité du monde jusqu'à l'absolu, et ils apporteront des exemples concrets à l'appui de leur affirmation. Pensons seulement au cas classique (au sens de pré-moderne) de l'Encyclopédie de Diderot et d'Alembert, qui certes systématise, mais sans prétendre tout expliquer. Comme l'écrit Marion Chottin dans sa contribution au présent dossier, dans ce contexte le système demeure ouvert et constitue l'ordonnancement de connaissances par une pensée libre et consciente de ses limites. Mais pensons également au $\mathrm{xx}^{\mathrm{e}}$ siècle allemand, qui, lui aussi, nous a légué au moins un débat important dont les protagonistes ont été le sociologue Niklas Luhmann et le philosophe Jürgen Habermas et dont l'enjeu était rien de moins que la pertinence d'un regard systématique sur la société ${ }^{2}$. Même si, aux yeux de certains, la réponse de Habermas semble l'avoir emporté, elle va de pair avec l'intégration par le discours de ce dernier d'une partie du vocabulaire luhmannien. Comme le souligne une des rares lectrices attentives de Luhmann en France, la politologue Estelle Ferrarese, « ainsi Habermas le premier va-t-il absorber, au fil de sa réflexion, beaucoup des prémisses de la sociologie systématique de Luhmann, auquel il emprunte tout simplement le concept de système ${ }^{3}$ ». En ce sens, l'importance du problème de la systématicité à la frontière de la sociologie et de la philosophie reste indéniable. Il est donc étonnant que le discours scientifique contemporain sur la mise en ordre des choses (qu'il vienne de la biologie, de la sociologie ou bien d'un autre domaine) puisse encore étonner le philosophe.

Il y a pourtant une autre raison plus profonde pour ma réaction: le langage des sciences fait écho à une préoccupation de certains philosophes, les mêmes (étrangement peut-être) qui ont poussé à la limite l'exigence de faire éclater les systèmes. Car la philosophie contemporaine a en définitive

2. Jürgen Habermas et Niklas Luhmann, Theorie der Gesellschaft oder Sozial Technologie: Was leistet die Systemforschung?, Francfort, Suhrkamp, 1971.

3. Niklas Luhmann, une introduction, Paris, Pocket/La Découverte, 2007, p. 233. 
été confrontée, alors même qu'elle poursuivait ses recherches, au besoin de réinventer la systématicité. Je n'évoquerai ici qu'un seul penseur dont on verrait bien, si l'on prenait le soin de le lire avec l'attention qu'il mérite, qu'il remet le problème à l'ordre du jour philosophique. Michel Foucault esquisse en 1984, l'année de sa mort, un dernier programme de travail: il l'appelle une ontologie critique et historique de nous-mêmes. Laissant de côté l'exégèse que réclame cette expression, je souhaite attirer l'attention sur les trois réquisits impliqués dans ce programme. Au moment d'expliquer en quoi il consiste, dans son texte «Qu'est-ce que les Lumières? », Foucault déclare ceci: «Ce travail a sa généralité, sa systématicité, son homogénéité et son enjeu ${ }^{4} »$. Sa systématicité, caractère qui est pour nous en question, touche à ce qu'il désigne successivement comme les «trois grands domaines » et les « trois axes »-le savoir, le pouvoir et l'éthique« dont il faut analyser la spécificité et l'intrication ${ }^{5}$ ». Ainsi, le retour de la philosophie anti-systématique (au sens philosophique du mot, justement) à la nécessité de décrire une expérience cohérente, recoupe jusqu'à un certain point le travail des sciences qui s'évertuent à constituer des systèmes et des sous-systèmes dans leurs domaines d'investigation.

À partir de cette coïncidence naît la question que je pose : qu'est-ce qu'être systématique aujourd'hui? Sans doute cette interrogation ne connaît-elle pas de réponse univoque. Être systématique en philosophie aujourd'hui est-ce la même chose qu'être systématique en science? Qu'entendent exactement les scientifiques par système? Et les philosophes? Cette préoccupation m'a rendu attentif à certains usages du mot faits par des scientifiques, après $\mathrm{M}$. Gros, par exemple son collègue au Collège de France, l'anthropologue Philippe Descola. Lors d'un exposé récent il employait le mot «système » pour décrire un " ensemble de phénomènes organisés ${ }^{6} »$. On peut ainsi, d'après lui, parler de la société comme d'un ensemble de systèmes indépendants: système politique, économique, juridique et autres. La structure, au sens lévi-straussien, n'est pas un système, ajoute-t-il, mais plutôt ce qui permet de comprendre les caractéristiques d'un système. Interrogé sur ce point, il explique qu'il fait du mot en question un usage « très empirique » et n'aspire pas à disposer

4. Dits et écrits, t. IV, Paris, Gallimard, édition dirigée par Daniel Defert et François Ewald, 1994, p. 575 .

5. Ibid., p. 576.

6. «Anthropologie réflexive et philosophie spontanée », exposé du 29/05/2009 à l'équipe Normes, sociétés, philosophies - NoSoPhi, Université Paris I-Panthéon Sorbonne. 


\section{Labyrinthe, $n^{\circ} 34$}

des schèmes qui rendraient cohérent l'ensemble d'un système. Il se sert plutôt de ce qu'il appelle une « épistémologie portative », étant toutefois convaincu que nul ne fait de la science sans croire qu'il y a des systèmes qui puissent être mis au jour. En cela, l'anthropologie rappelle le geste des philosophes qui souhaitent se conduire à l'instar des gens de science, comme Hegel, lorsqu'il déclare dans la préface de la Phénoménologie de l'Esprit que son propos « est de collaborer à ce que la philosophie se rapproche de la forme de la science - se rapproche du but, qui est de pouvoir se défaire de son nom d'amour du savoir et d'être savoir effectif ${ }^{7}$ ». De surcroît, la Phénoménologie de l'Esprit est présentée par son auteur comme constituant la première partie d'un Système de la science.

Mais les scientifiques ont le plus souvent voulu mettre un terme aux illusions philosophiques de tout embrasser, y compris lorsqu'ils se sont eux-mêmes laissés tenter par une autre sorte de méta-mise-en-ordre. À cet égard, Lévi-Strauss nous fournit un exemple éloquent. Dans l'Anthropologie structurale, il pose le problème de ce qu'il appelle l' « ordre des ordres », de la façon suivante:

Pour l'ethnologue, la société enveloppe un ensemble de structures correspondant à divers types d'ordres. Le système de parenté offre un moyen d'ordonner les individus selon certaines règles; l'organisation sociale en fournit un autre; les stratifications sociales ou économiques, un troisième. Toutes ces structures d'ordre peuvent être elles-mêmes ordonnées, à la condition de déceler quelles relations les unissent, et de quelle façon elles réagissent les unes sur les autres du point de vue synchronique. Ces tentatives pour formuler un modèle total d'une société donnée, confrontent l'ethnologue à une difficulté [...] : jusqu'à quel point la façon dont une société conçoit ses diverses structures d'ordre, et les relations qui les unissent, correspond-elle à la réalitée ${ }^{8}$ ?

Ce passage, dans lequel on voit la science à l'œuvre, appelle deux commentaires d'ordre philosophique. Premièrement, l'ordre des ordres n'est pas présenté comme un élément extérieur aux sous-ordres ou aux ordres partiels ou régionaux, comme un supplément qui viendrait clore

7. Georg Wilhelm Friedrich Hegel, Phénoménologie de l'Ésprit, Paris, traduction de Jean-Pierre Lefebvre, Aubier, 1991.

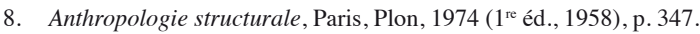


d'en haut un édifice qui autrement resterait inachevé. L'ordre des ordres n'est pas la clef de voûte anthropologique d'une construction ethnologique. Au contraire, si, comme le prétend Lévi-Strauss, il est un modèle total d'une société donnée, alors il s'apparente beaucoup plus à une sorte d'articulation entre les ordres régionaux, articulation dont le savant doit être en mesure de faire la cartographie ou de tracer le diagramme, avec les parties qui composent l'ensemble et leur disposition les unes par rapport aux autres. L'articulation rend, à un niveau total (c'est le mot de Lévi-Strauss dans le passage cité), l'ordre des sous-ordres qui réunissent des individus et des groupes dans la structure sociale. Il n'est rien de plus que cette articulation.

Une seconde remarque est la suivante: aussi ambitieux que semble ce projet, il ne comporte pas moins deux signes de modestie, comme deux limites que le scientifique s'impose à lui-même. D'une part, le modèle total concerne une société donnée et non l'ensemble des sociétés ni la société en général. La recherche ethnologique ainsi comprise n'est possible que dans le cadre de variations culturelles qu'elle n'abandonne pas, raison pour laquelle on pourrait dire qu'elle abandonne plutôt l'Homme, dont elle ne vise pas à constituer le modèle, mais justement, comme Lévi-Strauss l'écrivait ailleurs, à le défaire 9 . L'ordre des ordres est certes le modèle total d'une société donnée, il n'est pas le modèle de l'homme total. D'autre part, deuxième preuve de retenue, il n'est pas question pour l'ordre des ordres de saisir une société dans ses transformations historiques, mais seulement dans les relations synchroniques ou de simultanéité entre ses ordres régionaux. L'ethnologie et son effort de mise en ordre, ou de dévoilement de l'ordre, ne se proposent pas de saisir l'histoire d'un groupe, mais la façon d'être (hautement complexe) d'un groupe à un certain moment. Le modèle en question doit donc convenir à une société donnée, à un moment donné. Il doit pour ainsi dire rendre compte de l'état de choses total d'un groupe restreint. C'est dans ce sens que j'ai dit plus haut que le structuralisme a représenté aussi une critique des systèmes modernes de l'histoire totale ${ }^{10}$.

Tout cela est bien connu. Pourtant, il faut remarquer le fait suivant, aussi surprenant que révélateur: aussitôt esquissé ce programme et établis

9. La Pensée sauvage, Paris, Plon, 1962, p. 326.

10. D'autres détails sur l'ordre des ordres se trouvent dans l'Anthropologie structurale, op. cit., p. 363 sqq. 


\section{Labyrinthe, $n^{\circ} 34$}

la possibilité, voire l'intérêt, de retracer un ordre des ordres ethnologiques, Lévi-Strauss l'abandonne. En effet, pour un ethnologue, un scientifique attaché aux faits qu'il a lui-même observés et aux descriptions d'autres faits réalisées par ses pairs, la tâche semble démesurée. Encore fait-il allusion à un terrain déjà bien circonscrit, celui des études des phénomènes religieux, au sujet duquel il affirme: «Peut-être parviendronsnous à construire, en ethnologie religieuse, ces "modèles à petite échelle, destinés à l'analyse comparative... de variations concomitantes... tels qu'ils s'imposent dans toute recherche visant à l'explication des faits sociaux" [Nadel, 1952]. Cette méthode ne permettra de progresser que lentement; mais elle fournira des conclusions qui compteront parmi les mieux établies et les plus convaincantes, de celles que nous pouvons espérer en matière d'organisation sociale ${ }^{11} »$. Ainsi, on constate à la fois l'espoir et la réticence de Lévi-Strauss : espoir quant à la validité théorique de cette manière de procéder, contrebalancé, peut-être même contrecarré, par la difficulté pratique de la mettre en œuvre et de la mener à bien.

Cet aspect du comment et du pourquoi de l'abandon lévi-straussien du thème de l'ordre des ordres mériterait plus d'approfondissements. Dans cette première approche, $\mathrm{j}$ 'aimerais seulement suggérer comment nous serions capables de relancer ce défi sur un autre plan, que je me permettrai d'appeler philosophique et qui sans doute ferait frémir - ou simplement sourire - Lévi-Strauss, qui s'est toujours battu contre les spéculations sans base empirique. Je pars de deux constats ou de deux mots, le premier celui d'articulation, que je puise chez Ernesto Laclau et Chantal Mouffe, le second, celui d'agencement, qui porte désormais la marque de Deleuze et de Guattari. Comme l'explique Lévi-Strauss, l'ordre des ordres ne vise ni à la « restitution totale de la société concrète » qu'il s'était « d'abord efforcé d'analyser en structures » ni à l'affirmation « que, pour une société donnée, toutes les structures sont homologues, ce qui reviendrait à dire que chaque société constitue une sorte de monade, à la fois parfaitement cohérente et complètement fermée sur elle-même ${ }^{12}{ }^{\prime}$. Aussi le terme d'articulation semble-t-il convenir à ce but, trouver les relations par lesquelles les sous-ensembles à la fois sont réunis et interagissent les uns sur les autres, bref peuvent être ordonnés.

11. Claude Lévi-Strauss, Anthropologie structurale, Paris, Plon, 1974 [1 $1^{\mathrm{re}}$ éd., 1958], p. 349.

12. Ibid., p. 364. 
Depuis le début, j'attire l'attention sur le besoin contemporain de revenir à une certaine idée ou à une conception de l'ordre des choses, nécessité qui se manifeste plutôt dans la philosophie que dans les sciences, puisque celles-ci, de toute évidence, n'ont jamais abandonné le vocabulaire des systèmes et des sous-systèmes. Il n'en est pas de même pour la philosophie. Celle-ci a si puissamment érigé le système en but ultime de sa démarche; elle a cru avec tant d'énergie que le système pouvait être ce « tout sans fissure » dont parlait Alexis Philonenko dans une expression lumineuse pour désigner (reste à savoir si à tort ou à raison) la pensée kantienne ${ }^{13}$; elle a plus tard, dans une réaction de grande ampleur, fustigé si durement cette notion comme étant le symbole achevé du totalitarisme théorique et pratique, que toute tentative pour la rétablir aujourd'hui, ne serait-ce qu'au nom d'expériences, pourtant positives, du passé (comme l'Encyclopédie) serait vaine. En référence à l'hégélianisme et au marxisme (mais on pourrait penser à bien d'autres théories encore), Lyotard parlait des « grands récits », censés être capables de rendre raison de chaque détail et, dans cette mesure, comme des conceptions fermées sur elles-mêmes: ces « grands récits » philosophiques sont de nos jours discrédités ${ }^{14}$. Bien sûr, il faudrait se demander jusqu'à quel point la notion d'un système de pensées comme tout clos sur lui-même n'est pas une caricature des systèmes philosophiques effectivement proposés par la modernité à partir de Kant. Même dans le cas extrême de Hegel, on peut admettre avec JeanFrançois Kervégan qu' « en liant concept, vérité et circularité, la Préface [de la Phénoménologie de l'Esprit] suggère une conception dynamique du système, s'opposant à la représentation commune d'un achèvement du savoir. Le système n'est pas "un cercle qui repose clos en lui-même"; il est plutôt le "devenir de soi-même"15 ». Il n'en reste pas moins que la croyance philosophique dans le système est depuis longtemps brisée.

D'où la question: quel autre concept pourrions-nous mettre en avant qui satisfasse notre besoin d'une expérience articulée? À cette fin je souhaite proposer le terme d'agencement, non pas comme moyen pour combler un désir d'harmonie absolue, mais pour relever autrement le défi de la cohérence possible. Autrement dit, le terme retenu n'a aucune prétention à restaurer une situation définitivement révolue, mais il prétend relancer

13. «Introduction », dans Kant, Critique de la faculté de juger, Paris, Vrin, 1965, p. 15.

14. Voir Jean-François Lyotard, La Condition postmoderne. Rapport sur le savoir, Paris, Minuit, 1979.

15. Jean-François Kervégan, Hegel et l'hégélianisme, Paris, PUF, « Que sais-je ? », 2005, p. 47. 


\section{Labyrinthe, $n^{\circ} 34$}

autrement un principe d'ordre entre les mots, les gestes et les choses qui convienne à notre époque. Si nous avons aujourd'hui la conscience de vivre dans un temps post-systématique (post-métaphysique, dirait Habermas), temps de l'éparpillement et de la dispersion aussi bien dans l'arrangement disciplinaire (tout au mieux interdisciplinaire) des savoirs que dans le vécu quotidien, essayons de voir si notre époque ne pourrait pas se définir par elle-même, non pas comme post, mais bien plutôt comme époque à part entière: époque de l'agencement. Je me rends bien compte de l'ambition de cette entreprise, autant que de la faiblesse des moyens dont je dispose pour la mener à bien. Cependant, l'urgence de la tâche me pousse à rassembler quelques considérations sur la notion d'agencement, pour lesquelles je ne réclame que la valeur d'une première approche.

Premièrement, il conviendrait de noter que l'agencement se distingue d'autres formes connues de mise en ordre du divers, parmi elles le système, l'organisme et la structure. À la différence de ceux-ci, l'agencement est à la fois le résultat et le mouvement (ou l'opération) qui conduit au résultat, c'est-à-dire à l'état de choses. Il n'existe donc pas comme le système, lié à une systématisation, ni comme l'organisme, qui suppose une organisation, ni davantage comme la structure, précédée d'une structuration. Cette singularité de l'agencement est aussi ce qui le rend difficile à penser, car en lui le résultat et la procédure-mouvement se confondent, se recoupent, voire sont le même. L'agencement-résultat (l'agencement comme résultat) est plus qu'inséparable de l'agencement-procédure (la procédure d'agencement) ou de l'agencement-mouvement (le mouvement d'agencement): il coïncide avec eux. La difficulté se lie ici au fait que l'ordre nous apparaît simultanément comme mouvement, alors que souvent on dissocie les deux termes, allant même parfois jusqu'à les opposer.

Deuxièmement, il en découle que l'agencement est une forme de mise en ordre singulière par la façon dont il établit des rapports de nature multiple (nature sociale, épistémologique, politique) sous la forme d'une relation dynamique. Cela signifie qu'il ne prétend pas rendre compte de tout, et au cas où il le prétendrait il en serait incapable: en tant que relation dynamique, c'est-à-dire extérieurement et intérieurement, donc continuellement, en mouvement, il est en définitive incertain et incomplet. Il ne rend pas compte de tout, il n'embrasse pas tout, ni comme raison (ce qui est réel est rationnel, ce qui est rationnel est réel ${ }^{16}$ ) ni comme

16. Ibid., p. 16-19. 
« grand jeu qui soumettrait les antagonismes à son système de règles ${ }^{17}$ » (ou, sous une autre version, comme libre jeu des facultés) ni a fortiori comme système (tout sans fissure). De là, une conséquence: venant après l'époque du système, il convie à prendre congé de ce mot, sans pour autant abolir toute recherche d'une expérience cohérente. L'idée qu'il véhicule semble s'accorder avec les systèmes et sous-systèmes des scientifiques. De là aussi, un scrupule: comment se prémunir contre la tendance philosophique à chercher l'Agencement des agencements, le Système des sous-systèmes? Sans doute faudrait-il penser autre chose qui serait la « chaîne d'équivalence » ou encore l' « articulation » entre agencements ${ }^{18}$. Mais comment ne pas céder à la tentation de passer de l'articulation du multiple au système de l'ensemble?

Troisièmement, une autre conséquence de l'incertitude et de l'incomplétude des agencements mentionnées plus haut est le fait que, s'il est permis de reprendre ici le vocabulaire dont se servent Laclau et Mouffe pour parler des antagonismes, les agencements « ne sont pas des relations objectives, mais des relations qui révèlent les limites de toute objectivité $^{19} »$ ou, comme nous l'interprétons, les limites de l'être déterminé. Aussi ouvre-t-il sur le dehors, car il est, en partie du moins, au dehors et il insuffle du dehors dans le dedans, brouillant du même coup ce partage. Cela signifie qu'il existe, dans l'agencement, un surplus du mouvement sur l'ordre ou de la transformation sur la forme et que ce qu'il y a de dehors dans l'agencement empêche la stabilisation et la clôture de ce dernier sur lui-même, s'opposant à son autarcie et à sa pleine effectivation. Il faudrait se demander s'il n'y a pas, dans cette idée d'un dehors de l'objectivité, une dimension de lutte qui échappe à toute systématisation, pour autant que la lutte échappe au système, ne se laissant pas saisir par lui comme l'un de ses éléments ou de ses mouvements internes ou intrinsèques. La «lutte contre le système » apparaîtrait alors moins comme un mot d'ordre que comme une force («la lutte ») s'opposant («contre») au fonctionnement omnipotent du système.

Quatrièmement, l'agencement existe là où il n'y a pas d'acceptation totale d'une réalité donnée, c'est-à-dire d'une réalité présente. En étant

17. Le mot est d'Ernesto Laclau et Chantal Mouffe dans leur livre Hégémonie et stratégie socialiste. Vers une politique démocratique radicale, trad. de Julien Abriel, Besançon, Les Solitaires intempestifs, 2009, p. 27.

18. Ibid., p. 34-35.

19. Ibid., p. 27. 


\section{Labyrinthe, $n^{\circ} 34$}

une relation dynamique, l'agencement ne concerne pas ce qui est présentétant-là, mais plutôt ce qui est actuel ou en acte, raison pour laquelle il est toujours un rapport d'actualité. Le mot même d'agencement est retenu ici par les résonances qu'il provoque, sans toutefois renvoyer à une étymologie commune : agencement, agencer, s'agencer, agent, agir, action, acte, actualité ${ }^{20}$. L'action permet de dissoudre les prestiges de l'identité comme propriété des individus, l'identité étant à comprendre ici, avant tout, comme la présence de moi à moi et de moi aux autres.

20. Sur certains de ces mots et leurs rapports, voir Étienne Balibar et Sandra Laugier, « Agency », dans Barbara Cassin (org.), Vocabulaire européen des philosophies, Paris, Seuil/Le Robert, 2004, p. 26-32. On remarquera néanmoins que le mot agencement n'est pas traité dans cette entrée de dictionnaire. 